\title{
Experiences with the Development of a Portable Network Operating System
}

\author{
U. Hollberg, B. Mattes, A.Schill, H. Schmutz, \\ B. Schoener, R. Staroste, W. Stoll \\ University of Karlsruhe \\ Kaiserstraße 12 \\ D.7500 Karlsruhe \\ IBM European Networking Center \\ Tiergartenstraße 15 \\ D-6900 Heidelberg
}

\section{Introduction}

In this paper we report on the experience gained during the development of the Distributed Academic Computing (DAC) Network Operating System (NOS). DAC NOS is the result of a joint effort between the University of Karlsruhe and the IBM European Networking Center at Heidelberg.

The original idea behind the DAC project was to bring together university and industry research to address system problems arising in the upcoming university environment, which may be characterized by many autonomous computers ranging from workstations to large supercomputers, which are interconnected via high speed local area networks. As a consequence of the autonomy and, of course, the different size of computers, it appears unlikely that these computers would be serviced by a single type of operating system. The reality is a multi-vendor environment, which is likely to stay for the foreseeable future. 


\section{Resource Sharing}

With this background we determined the absence of resource sharing between heterogeneous computers as a central problem deserving a systematic solution. Users should be able to utilize data, programs, compute power and peripheral devices in the network from within their own environment and without having to learn the rules of communication with a - from their point of view - alien operating system. On the other hand, the owners of resources should be free in their decision to whom and to what extent they offer part of their services and resources. In particular we set ourselves the following objectives:

- The native user interfaces of the participating Operating Systems should remain unchanged. Applications of the native system should continue to run unmodified (Coexistence).

- Users should be able to access remote resources from their own native environment without having to learn anything about the remote system. Ideally, the users would access remote resources in the same way as local resources (Access Transparency). For example, the user of system A should be able to edit a file from remote system $B$ with the native editor of system $A$.

- Node autonomy should not be affected. It should be easy for the owner of a node to grant access to selected remote users. It should be at least equally easy to prevent access from unauthorized users. Clearly, in an environment with open access to computing equipment the question of identifying accessible resources, of authorization, of resource management and of accounting need to be solved systematically in a way which preserves node autonomy.

- The developed solution should be integrated with local systems in a way which reduces the complexity of portation of the system to another system (Portability).

- There should be no dependency on specific underlying network architectures and protocols. Concepts and Services for the transport of messages should be based on open, public network architectures. To achieve a maximum of connectivity, these services should be offered independent of the underlying network architecture.

\section{The DAC Network Operating System}

In order to meet the above objectives we had to design a solution, which may be characterized as Network Operating System although our objectives clearly go beyond earlier attempts in this direction.

The DAC NOS has conceptually a horizontal and a vertical structure. Horizontally it is a completely connected graph of Logical Nodes, the units of distribution. The developer of DAC NOS for a specific operating system has to make a decision on how to map logical nodes to real objects. Often, a logical node will be mapped to a heavy weight process, i.e. a process with its own address space. However, more importantly a logical Node should correspond to that part of the system, which is typically assigned to a single user. 

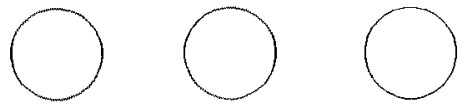

\section{Applications}

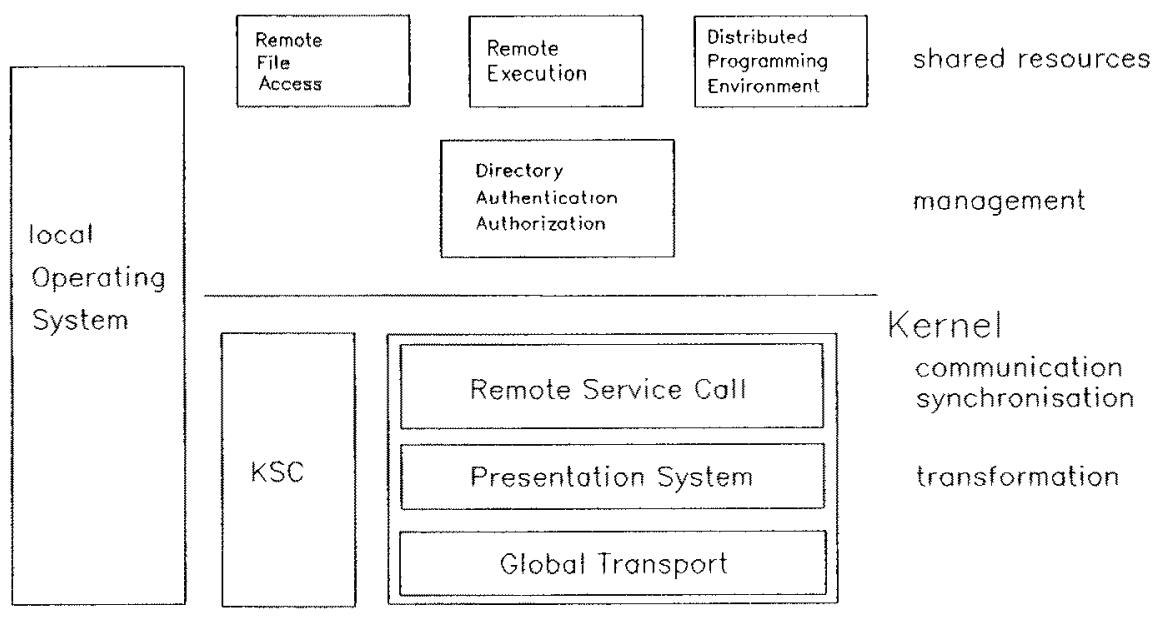

Figure 1.1 The Components of the DAC Network Operating System

Figure 1.1 shows the vertical structure of DAC NOS. Within a single logical node the application programs coexistently have access to the local operating system and to the Network Operating System extension. The NOS consists of the Kernel and of System Services.

The Kernel is "resident" in each logical node. It consists of four components. At the lower level it provides a portability environment, which will be discussed in section 2. The upper layer of the Kernel contains the protocol machine and the translator for data presentation. This is the complex part of the Kernel, which was designed to be portable. The upper layer of the NOS Kernel will be discussed in section 3.

System Services are implemented as applications of the NOS Kernel. A service is a distributed application consisting of clients ( resident in the using logical node) and of one or more servers resident, in general, in dedicated logical nodes. As pointed out before, our goal was to develop a system which makes it easy for users to share resources while maintaining node autonomy. Directory, Authentication and Authorization Services play therefore a central role in DAC NOS. Section 4 will be devoted to these services. Finally, section 5 will give a report on the design and implementation of a complex network application built on top of the NOS Kernel and the Directory. This application was developed in parallel with the NOS-Kernel and thus provided useful feedback during early stages of the project. 


\section{The Portability Environment}

The aim to develop the Network Operating System (NOS) Kernel such that it could be ported to different machines with limited expense clearly was motivated by the software costs. Therefore when starting the project it was natural to worry about

- how to support designers with adequate constructs to structure the software, and

- how to increase the portability of the developed code.

\subsection{Requirements}

\section{Functionality}

To support the development of complex protocols one needs suitable mechanisms to separate independent parts. Rather than develop a software system design using top-down decomposition, it has been suggested [1] that a better design methodology is decomposition of functions into a set of concurrent processes that can run independently of each other. They are particularly useful for layered architectures where it is important to facilitate the handling of asynchronous events which may be produced for example by device drivers or timeouts necessary in communication protocols. To this end, heavy weight processes, i.e. processes with their own address space, are not useful. Required are light weight processes which are characterized by shared memory and efficient inter-process communication. The light weight process is the central object in such an environment but in addition a set of objects and operations is necessary in support of communication, of synchronization and of higher level objects.

The introduction of the NOS should minimize the burden to the user and preserve his customary environment concerning both, the Local Operating System (LOS) interface and the use of existing applications. The coexistence of the NOS with an accustomed environment is necessary to obtain the desirable acceptance. Coexistence means that the NOS extensions run concurrently with the applications : As an example, consider the editing of a remote file. In this case the editor should be unaware of the remoteness and communication with the remote file server should be completely transparent and parallel to the editor.

The absence of light weight processes in some of today's operating systems and the requirement that the introduction of the NOS should not affect existing applications and not necessarily be visible to the user led to the idea to develop the Kernel Service Call (KSC) component [2].

In addition, the transport service is part of the portability environment. It should offer a simple send/receive interface based on a global addressing scheme in a way which hides the underlaying network 
architecture and it should provide for a maximum of network connectivity. The component covering this requirement is called Global Transport [3].

\section{Portability requirements}

To reach a high degree of portability for the NOS we had to choose an implementation language which supports portation of developed code. It was obvious that the Kernel Service Call component was suitable not only to expand the LOS but also to offer a host independent interface to necessary LOS services, e.g. storage management and the timer support.

Portability requires an implementation language that is widely available. Moreover the language should be reasonably well standardized avoiding differences in dialects, machine dependencies, semantic and syntactic aberrations. For our needs there were two additional requirements which had to be satisfied by the language. It should facilitate system programming and it should be suitable for the development of system services and applications above the NOS.

Figure 2.1 shows the structure of the Portability Environment and points out that our implementation language is " $\mathrm{C}$ ".

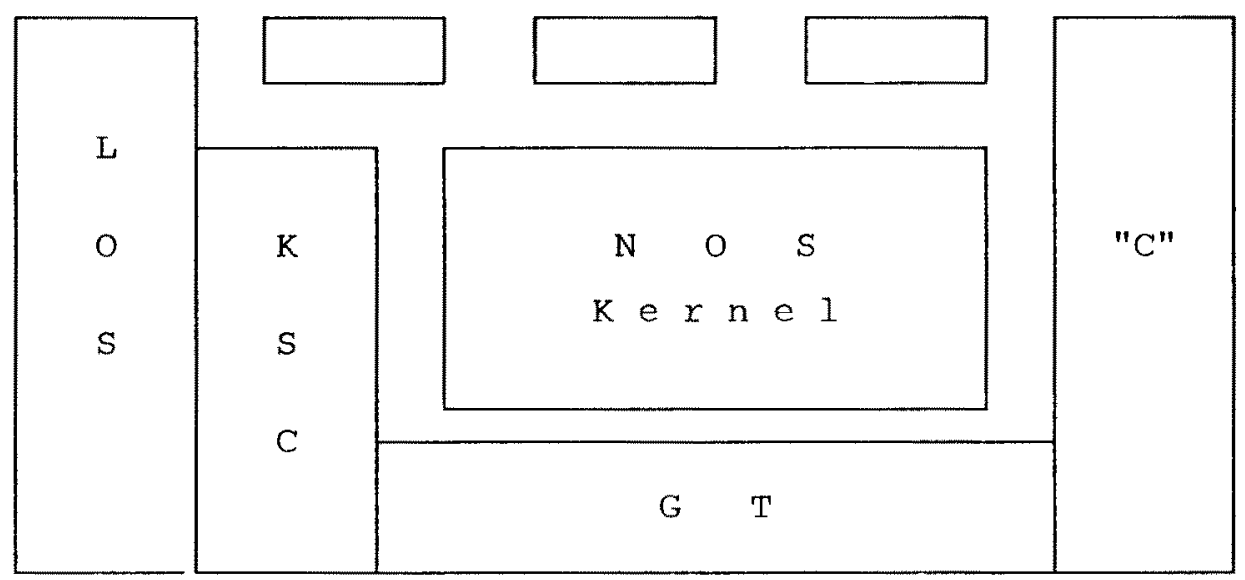

Figure 2, 1 The Portability Environment

\subsection{The Implementation Language}

One of our first decision in the project was to use " $\mathrm{C}^{\prime}$ as our implementation language. Wide spread support of the language is a necessary prerequisite to obtain portability. The only alternative considered was PASCAL. The decision in favour of " $\mathrm{C}^{\prime \prime}$ was triggered by existing experiences with PASCAL and the necessity to implement efficient system programs. 


\section{Experiences with " $\mathrm{C} "$}

We do not feels enthusiastic about " $\mathrm{C}$ ". However it is the best choice for our needs. Programs written in " $\mathrm{C}$ " are portable to a high degree with the exception of two problem areas.

- The runtime environment is not subject of the language standard. There may be different names for the same function or different numbers or types of function parameters. In the worst case some functions do not exist at all in a certain environment.

- It is easy to declare and use nonportable data structures, e.g. the order of bit fields and their boundaries is non-standard, only primitive access to bit fields guarantees portability. Unions used to overlay different data structures on the same storage area have to be considered carefully, since different lengths of their substructures on different machines could make them nonportable.

Some problems arrived through deviations between compilers on different machines sometimes even with the use of a new version of a compiler. Just to give an idea, a list of possible aberrations follows :

- The maximum length of names for preprocessor-, local- or external variables (hopefully the compiler warns you when detecting a duplicate definition).

- Default types - an integer may be a short integer or a long integer.

- Sign extension - for example on shift operations.

- Automatic type conversion which is associated with the last item.

Last but not least and most exciting, since we did not expect it, we discovered some simple bugs in the compilers.

\section{Implications}

From all of this experiences with " $\mathrm{C}$ " we derived two implications. First we defined a set of additional conventions to be obeyed by implementers of system components. They are summarized in a document [4]. Second there is a constant need for better compilers with cross module parameter checking and extended support for symbolic debugging. In addition the code generated by the compilers is not very sophisticated, it leaves ample room for optimization. Since " $\mathrm{C}$ " is more and more accepted as a standard for portable system programs, we look forward to a new generations of improved " $\mathrm{C}$ " compilers.

\subsection{Kernel Service Call}

The Kernel Service Call expands the capability of an existing local operating system by light weight processes and services considered mandatory for the synchronization and communication of parallel processes. It should be noted that KSC primitives are strictly local and do not solve the problems of heterogeneity neither for synchronization of processes on different logical nodes nor for data presentation. 


\section{KSC objects and operations}

The one active object in KSC is the process which acts on other KSC objects by executing their associated operations. Due to its nature the process descriptor varies between $\mathrm{KSC}$ instances on different machines. Although the process descriptor supports a name field, a KSC process is identified by the address of its descriptor to increase efficiency of process management .

Interprocess communication has to be expressed with Port, Carrier and Notice objects. Carrier and notice are types of messages. A carrier implements a two-way-message which can be returned by the receiving process and be waited upon by the sender, whilst notices represent one-way-messages. A port is a message queue, multiple processes may wait on a port. Figure 2.2 shows two scenarios of inter process communication. In the first example the calling process (client) needs a certain service from the called one (server). Therefore he specifies the request in the message part of a carrier and sends it to a port, which is served by the called process. The client continues execution of its program until it reaches a point where the results from the server have to be available and consequently the client waits on return of the carrier. Meanwhile the server waiting on the port got access to the carrier. It then evaluates the required results, returns them with the carrier and ends its cycle by waiting again on the port for incoming requests.

Calling Process
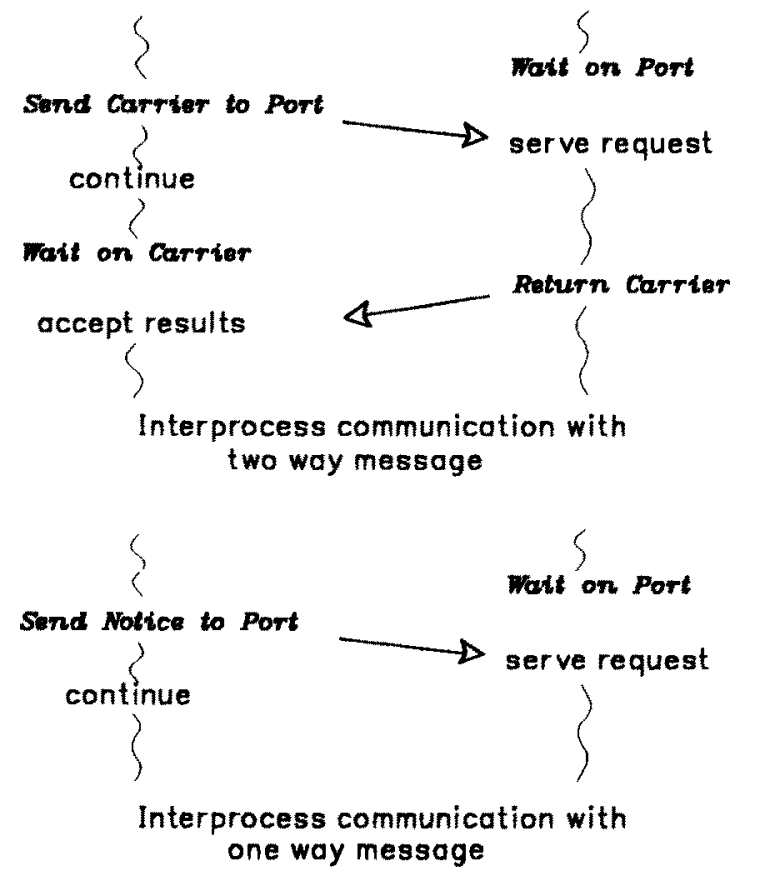
The second scenario may be suitable for device drivers where the calling process notifies the called process of a certain event. It continues without taking care about future life of its information. The called process works on the data specified with the notice and then is free to either delete the notice or to reuse it.

The Signal may be used for locking mechanisms. Carrier, port and signal have an event property. To support disjunctive multiple wait KSC introduces an Event List object which contains a list of the individual event type objects. Figure 2.3 summarizes the KSC objects and their semantics.

\begin{tabular}{|c|l|}
\hline process & $\begin{array}{l}\text { the active object in KSC, executes kSC } \\
\text { operations on the other objects }\end{array}$ \\
\hline carrier & $\begin{array}{l}\text { message to request a certain service from } \\
\text { process, has to ba sent to port and may } \\
\text { be returned to waiting sender }\end{array}$ \\
\hline notice & $\begin{array}{l}\text { one-way message to request a cortain service } \\
\text { from process, has to be sent to port }\end{array}$ \\
\hline port & $\begin{array}{l}\text { aveuss incoming carriers or notices which must } \\
\text { be served by a process waiting on the port }\end{array}$ \\
\hline signal & $\begin{array}{l}\text { set of objects which can be waited upon, } \\
\text { serves as ksc multi wait operation } \\
\text { indicates to a process the occurrence of an }\end{array}$ \\
\hline
\end{tabular}

\section{Figure 2.3 The Kernel Service Call objects}

\section{Experiences}

The use of KSC primitives in the NOS kernel, in Global Transport and in early versions of other system components has served to increase our confidence in their utility and completeness. Only a few operations, particularly for extended process management support, have been added later to meet requests from KSC users. So in our experience KSC offers the right set of primitives to facilitate the development of complex protocols. 
KSC has first been implemented under VM/CMS. The first question when porting KSC to another operating system is whether the LOS supports light weight processes or not. If so, KSC processes can be mapped to their equivalent in the LOS which, in general, would remove the requirement to establish a KSC dispatcher. This has been done on the two systems to which KSC has been ported. For PC DOS, the CP88 extension [5] has been used as an efficient dispatcher. For VAX VMS heavy weight processes with segment sharing have been used.

Most of the code necessary to execute KSC operations has been implemented in " $\mathrm{C}$ ", therefore this part of $\mathrm{KSC}$ is portable to a high degree and merely has to be recompiled on target machines. We stripped machine dependent parts into separate files to facilitate their replacement and to obtain a manageable system.

The original implementation of KSC consists of 7000 lines of code from which about $50 \%$ are written in C. This was done in roughly two person years. Portation to the VAX VMS or the PC DOS environment has been done in nine respectively six person months.

To evaluate the performance of KSC we measured a simple turn around cycle (send-wait, wait-return) as depicted in the first scenario of Figure 2.2. Since the actual processor time was not available on each machine we used the elapsed time to obtain comparable results listed in Figure 2.4. It is important to note that on VM/CMS and VAX VMS measurements have been done during normal daytime with the overhead produced by other users on the system. Therefore the actual processor time for a turn around cycle of $0.25 \mathrm{msec}$ on the VM/CMS is a more relevant value.

\begin{tabular}{|ll|}
\hline Configuration & alapsed time for a turn around cycle \\
Wh / CHS on 4361 & $0.6-0.9 \mathrm{msec}$ \\
VAX WHS on 8600 & $1.5 \mathrm{msec}$ \\
PC DOS on PC AT & $2.5 \mathrm{msec}$ \\
Figure 2.4 KSC performance data
\end{tabular}

\subsection{Global Transport}

The Global Transport (GT) system is required to support connectivity between users on logical nodes in the network. In order to provide an interface where the user does not need to worry about details of connection handling the Global Transport offers a simple send-receive interface. Therefore it shares two KSC port objects with its users. One "sendport" for transmitting messages and one "receiveport" for waiting an incoming messages. Thus a GT user first builds a carrier which contains information describing the data to be transmitted and the address. The GT user sends the carrier to the "sendport". The carrier is then accepted by GT and returned to the sender indicating that GT now is responsible for data delivery. The other GT user waits on the "receiveport" at the remote site and obtains an indication 
that data are available. He then accepts the data. For the sender there is a high probability, that the message will be delivered but no guarantee. We call this type of service Reliable Datagram .

Actually transport of data happens over different communication media (LANs, WANs). To provide the required transparency to the GT users, the system must hide the underlaying differences and integrate the heterogeneous subnets into a global network.

To identify partners in the network a unique address has to be assigned to each logical node in the network. In our current implementation we use the TCP/IP addressing scheme. In the future we will use OSI transport protocol addressing scheme with human readable addresses.

\section{Global Transport Configuration}

Figure 2.5 shows the current configuration of the Global Transport and the different subnetworks that have been integrated. It also points out that the Global Transport uses the most efficient communication media in each local environment where "local" means a set of logical nodes residing on a LAN or inside a real machine.

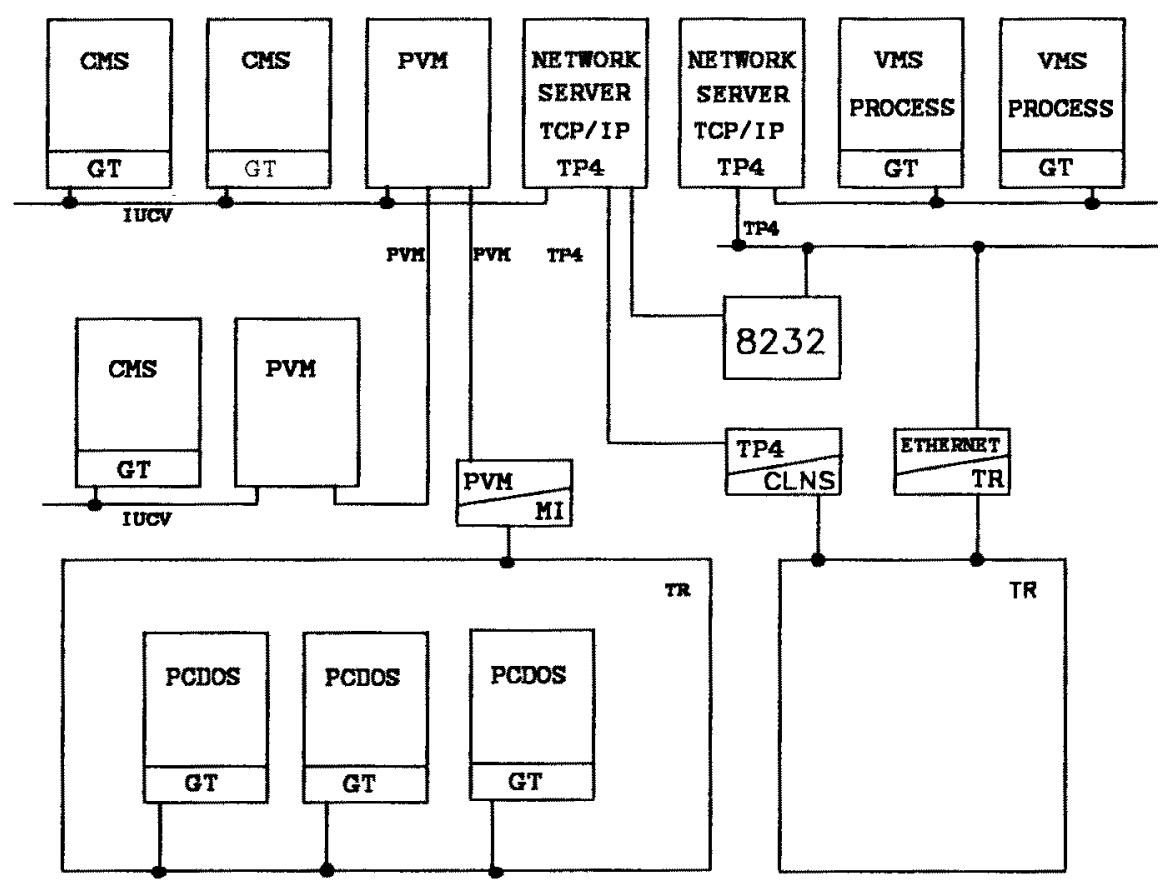

Figure 2.5 Global Transport configuration 


\section{Experiences}

In the early days of the project we felt that reliable datagram serves as an adequate interface for the NOS Kernel. With progress of the project we feel now that connection oriented transport services also could be adequate. Although the simplicity of using the reliable datagram and its independence from different connection services are still considered as very positive points of our solution.

The first implementation of Global Transport under VM/CMS encompasses 6500 lines of code and required one person year. A lot of work has since been spent on the DEC-IBM link and on the PC Version of the Global Transport. This includes the use of special hardware and their integration. Once more it has been shown, that the use of already tested products is the better, and often also the more efficient solution.

Detailed performance measurements are planned. But the overhead of GT is included in the NOS Kernel performance data shown later on.

\section{The NOS Kernel}

The upper layer of the DAC NOS Kernel consists of the Remote Service Call (RSC) component and the Presentation System. RSC provides an uniform interface for the implementation of distributed applications in a heterogeneous environment. The Kernel is based on the Client/Server model of cooperation and is responsible for providing the network transparent interprogram communication. The presentation system ensures that applications see the data in the respective local representation.

In this chapter we briefly describe RSC and some of its significant properties followed by report on the experiences during development and the achicved results.

\subsection{Requivements and Objectives}

A major constraint in the design was to ensure that the NOS will coexist with the LOS and its existing utilities and applications. Thus the NOS will not replace existing services and applications but act as an extension for supporting distributed applications. Coexistence in a Network Operating System is mandatory for achieving network transparency.

The basic objective of RSC is to provide operating system level primitives for a distributed heterogeneous environment which are functionally equivalent to those of local operating systems. This is an ambitious objective primarily because communications in local system relies on the ability of sharing memory between communicating partners. The Kernel of local system makes heavy use of shared memory for protection, authorization, accounting and recovery, to name some major system provided functions. A proper substitute for the role of shared memory has therefore to be found. The cooperating partners in the network must keep their functional and administrative autonomy. The autonomy 
aspect implies that the NOS Kernel must be more than just interprocess communication and synchronization. An integral part of the Kernel must be access protection of objects against unauthorized usage. As in a local system the NOS Kernel must offer means required for charging a requestor of a service for the work that was done on his behalf without placing more burden on application programs than on a local system.

To support the development of distributed applications efficiently, several transparency requirements have to be met. The Kernel services have to be provided independent of the location of the objects, on which operations are performed. This is location transparency. In a heterogeneous environment there may be different internal data representations on the individual nodes. To keep a transparent view on the data the required data conversions have to be performed transparently to the application. This term is referred as presentation transparency.

\subsection{Distributed Object Sharing with Protection}

The major guideline for the NOS Kernel design was Object Sharing. In the local system environment shared memory is known to be a very powerful way of communication. However, extending the notion of shared memory to the NOS would violate the autonomy requirement. Consider the case of a large network in which every NOS Kernel on a workstation has access to all of the addressable memory in the network. To substitute for the shared memory, access controlled object sharing was found as the best possible solution in the presence of distrust between logical nodes. From a RSC-application point of view, objects reside in a single global object space. Logical nodes need first to obtain access rights before they actually can perform operations on an object. Figure 3.1 illustrates this concept. An RSC object may be shared by multiple nodes only if each accessing node has the access right for the object. The absence of shared memory in distributed systems has been noticed as a major inconvenience for the development of applications $[6,7]$. Object sharing overcomes this inconvenience.

\subsection{RSC Objects and Operations}

The object model is an appropriate vehicle in a distributed environment [8]. It offers an uniform coherent view of distributed objects where the distributed reality of their implementation, the heterogeneity, communication, naming and protection are completely transparent to the user. In addition there is a set of allowed operations with parameters and a set of rules governing the legal sequences of operations and conventions for specifying operations and parameters. Resources may move in the network without reflecting the applications using them. The object model in the NOS Kernel is the basis for achieving the required network transparency and supporting the development of distributed applications.

The NOS Kernel offers objects comparable to the level of objects offered by a local operating system Kernel. Combined with the appropriate operations these objects provide a consistent base for building 


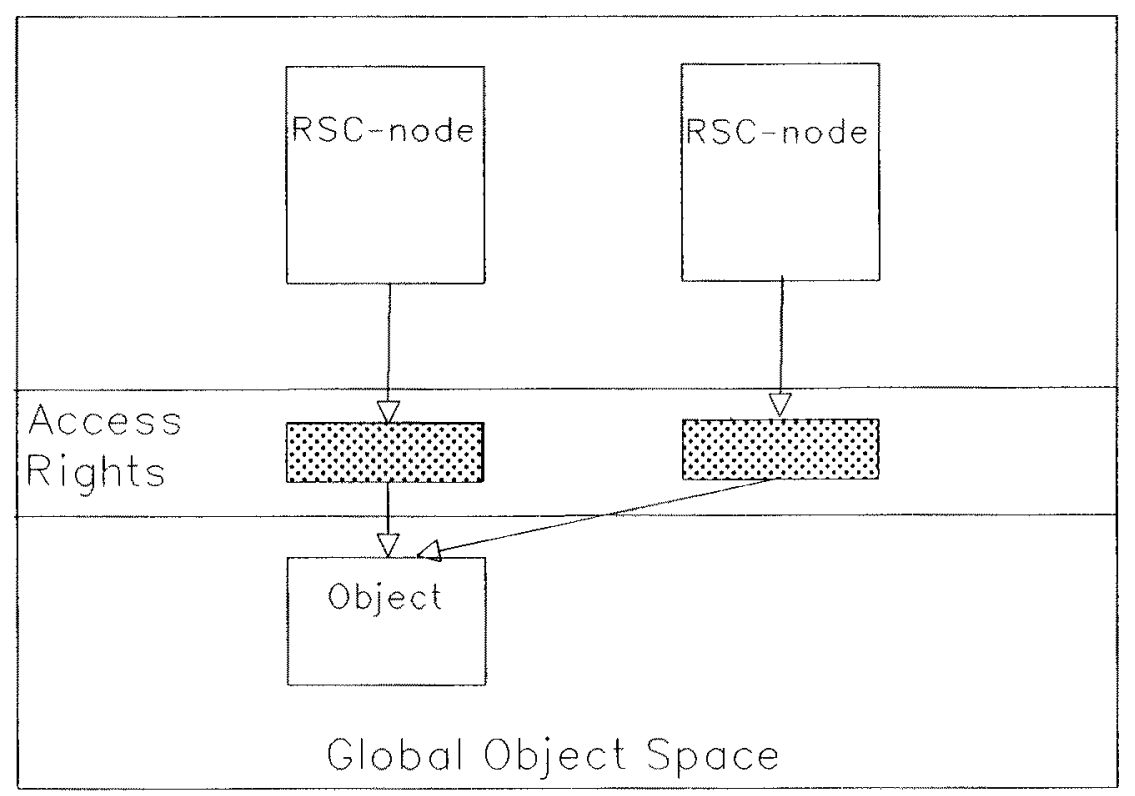

\section{Figure 3.1 Object Sharing with Protection}

higher level objects like files, procedures, mail boxes and network processes. In addition, the model is open for extensions. New objects and services can easily be added.

The set of RSC objects and their major characteristics are described in Figure 3.2. The column "event" indicates if and how the corresponding object can be waited upon. The column "sharing" expresses the relation of the object and the number of possible sharers.

\begin{tabular}{|c|c|c|c|}
\hline object & Function & Sharing & Event \\
\hline $\begin{array}{l}\text { Process } \\
\text { Port } \\
\text { Carrier } \\
\text { Notice } \\
\text { Window } \\
\text { Account } \\
\text { Lock } \\
\text { Event-list }\end{array}$ & $\begin{array}{l}\text { issues operations on objects } \\
\text { is a queue for service requests } \\
\text { multiple clients may concurrentiy } \\
\text { request the same service, multiple } \\
\text { servers may provide the service } \\
\text { passes a service request with } \\
\text { temporary access rights to a server } \\
\text { is a one-way message } \\
\text { is used to grant access to virtual } \\
\text { memory information about resource } \\
\text { keeps inford it } \\
\text { usage and dispatching parameters, it } \\
\text { identifies clients to servers in } \\
\text { processes and carriers } \\
\text { provides synchronization of } \\
\text { processes } \\
\text { supports wait on a collection } \\
\text { of events }\end{array}$ & $\begin{array}{l}1: 1 \\
1: n \\
1: n\end{array}$ & $\begin{array}{l}\text { Cāil arrived } \\
\text { end of call } \\
- \\
- \\
\text { complete } \\
1 \text { event complete }\end{array}$ \\
\hline
\end{tabular}


The set of operations operating on the RSC objects can be grouped into several classes: object management, process management, event handling, object sharing and service binding, request processing, message sending, data presentation, exception handling, and debugging support. RSC operations are provided as library routines. Some of the RSC operations are described at more detail subsequently. A complete description of all operations is in [9].

\subsection{Properties of the NOS Kernel}

\section{Object Sharing}

Access rights to sharable objects can be passed in two different ways:

- explicitly by OFFER/SHARE operations; the OFFER operation determines the access rights of the sharers. This represents a long-term binding between clients and a server.

- implicitly in a Service Call for the duration of the call. The caller specifies the access rights of each object passed to the callee. This can be called short-term binding since all access rights are lost after the request has been returned.

The access point for a service call is the Port object. A port is a queue which can be accessed by multiple clients and served by multiple servers.

The explicit sharing can be illustrated by a small example where a server creates a port and offers the port with his servicename and the access rights for the possible sharers to the public of clients. A client can request services from the port after a successful SHARE operation with the servicename and the access rights.

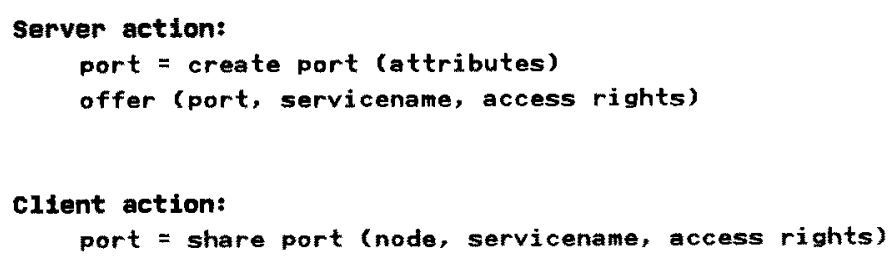

Implicit object sharing takes place during a service call with the Carrier object. Figure 3.3 is an example of a simple request. After a successful share operation the client creates a carrier object, places access rights to objects and an optional message into the carrier and sends the carrier to the port. The server receives the carrier, gets the access rights, performs the required service, returns the carrier after completion thereby loosing the access rights contained in the carrier. 


\begin{tabular}{|c|c|c|}
\hline Client & NOS & Server \\
\hline $\begin{array}{l}\text { prt=share (node, 'DBSERV', SEND) } \\
\text { crrl-create carrier (attrib) } \\
\text { place access rights into crr1 } \\
\text { send carrier (crr1, prt) } \\
\text { (continue to work) }\end{array}$ & $\rightarrow$ & $\begin{array}{l}\text { prt1=create port (attr) } \\
\text { offer (prt1,'DBSERV',SEND) } \\
\text { wait (prt1) } \\
\text { get carrier (crr1) } \\
\text { get access rights } \\
\text { perform service, } \\
\text { use access rights }\end{array}$ \\
\hline $\begin{array}{l}\text { wait carrier (crr1) } \\
\text { obtain results }\end{array}$ & $<$ & return carrier $(\operatorname{crr} 1)$ \\
\hline (continue program) & & \\
\hline
\end{tabular}

Figure 3.3 Service Request Cycle Scenario

In abnormal situations a client may wish to cancel a service call. He can retract the carrier and thus retract the access rights at any time by means of the retract and delete operations. Note that the objects involved by a service call need not reside at the server's or client's site. They may reside at some node, the access rights may have been passed to the client or server by a previous service call or a share operation.

An interesting property of the object sharing is that an object can be passed as parameter. The callee may use the object in the same way as the caller. This parameter passing by reference is an unique RSC feature which distinguishes it from the usual RPC based approaches. The situation is illustrated in Figure 3.4. The call in procedure $C$ will act on $A$ ( a procedure or object local to procedure $M$ ) independent of the relative locations of $\mathrm{M}, \mathrm{B}$, and $\mathrm{C}$. Access rights can bc passed along in the network just like pointers in a local system, but with protection. Only passed pointer are valid. 


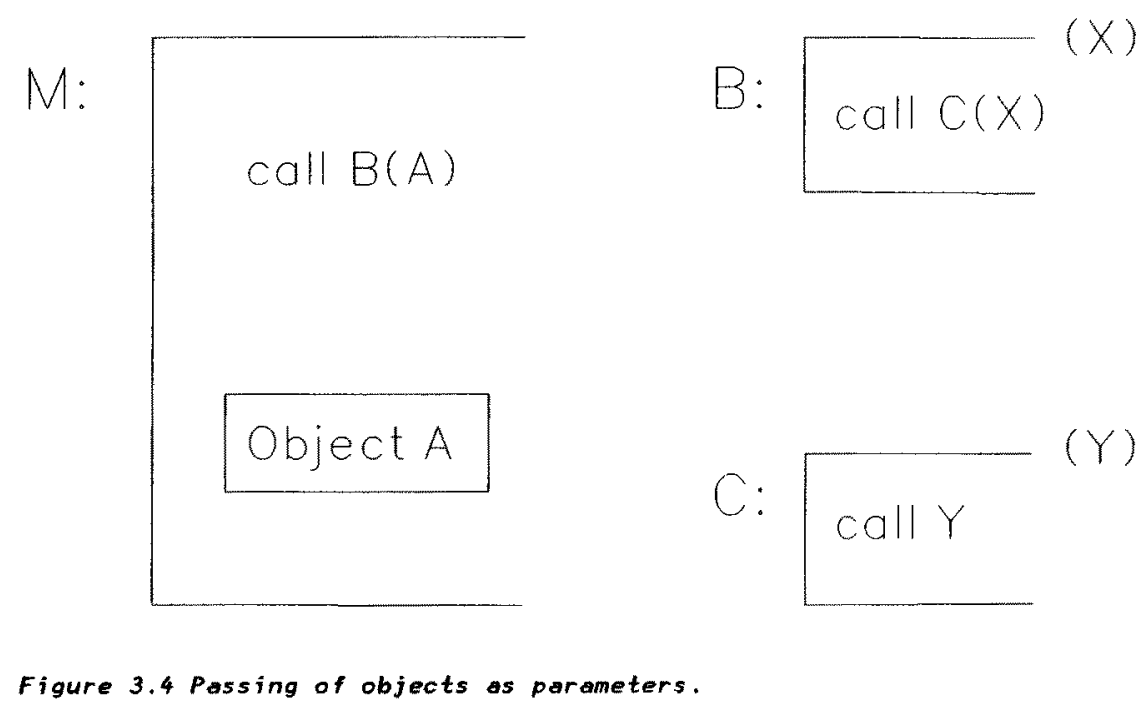

\section{Asynchronous Interaction}

RSC supports asynchronous cooperation with respect to the client and server behavior. A client may start new service calls before the previous calls have been completed, a server may accept new requests before he has processed all previous requests. Again, the local communication mechanism in operating systems are the model for remote communication, e.g. the $1 / 0$ instruction together with the interrupt mechanism. A service requestor can issue several requests in "parallel" and has control over each individual request with an explicit WAIT operation. For building complex distributed applications there is a need for asynchronous communication.

The process may also wait for several events at the same time. The Event-List object is able to hold several event-objects and provides the "multiple wait" facility. If one event in the event-list becomes complete the waiting process is resumed.

\section{Presentation System}

Data objects in RSC, e.g. a storage window or a carrier message may have an associated Format Description. If so, the NOS Kernel ensures that data are presented in the proper local form to application programs. In general, format descriptions are associated with data objects through the creation of typed ports. Data conversion during calls to typed ports is completely transparent to the caller. In this way RSC offers presentation transparency in a heterogeneous network.

Format descriptions are specified in the language NATRAS. A NATRAS compiler produces an internal representation of the Format Description which may be attached to data objects and which is passed between logical nodes when the need arises to perform conversion at both ends. 


\section{Accounting}

In a local system a service will usually be charged to the account of the user who had requested the service. A LOS kernel also keeps process state and system information in control blocks. This information identifies the user and is used by system services like dispatching, authorization, resource management and accounting. The RSC Account object extends this concept for the distributed environment. Each process is associated with an account object. Thus the NOS is aware at any time who is to be charged for a service. When preparing a service call, access rights to the account object of the requestor will be automatically transferred in the carrier to the server. The server shares the account object of the client during the service call and accumulates accounting, dispatching and system information into the client's account. Similar to local system facilities the user need not be aware of this mechanism.

The account object uniquely identifies a service requestor and can be used to support fine grain authorization. A server may restrict a service to a certain number of users, though much more users share the server's port. The server inherits the account of the requestor. By access to its current account object the server determines, on whose behalf it is running and can thus decide on fine grain authorization. The account object of a requestor may also be passed to other nodes, e.g. subservers if they are involved in the service call. Furthermore the RSC account object can be used as a basis for network wide scheduling, resource control and monitoring. Note that RSC itself does not provides these services but delivers the raw data and hooks for collecting these data.

\subsection{Experiences}

The RSC prototype is currently running in a network of IBM VM/CMS, DEC VAX VMS and IBM PC DOS computers and used by three research groups. Subsequently we will report about the experience in porting the NOS Kernel and give some performance data.

\section{Portability}

The RSC prototype was first developed on the IBM VM/CMS system and then ported to the DEC VAX VMS and IBM PC systems. The VM/CMS system is the development-system and base. It stores all relevant RSC data and programs.

Since the lower level components of the Kernel, KSC and GT, were designed to provide a system independent interface for use by RSC, RSC is portable. Nevertheless, some small parts of RSC like initialization and shutdown are system dependent functions and had to be implemented on each individual system.

The source code of the NOS Kernel is maintained at a single base for multiple target systems. The system dependent source code and data structures are contained in so-called dependent files. The dependent files hide differences in data structures, e.g. alignment and bit fields, and during the download of the code from the master system to the target system only the relevant dependent files will be 
downloaded. A further possibility to circumvent system dependencies in $\mathrm{C}$ is the conditional compile feature '\#ifdef'. Small fractions of the RSC code use this feature.

The major porting effort was to develop tools like download procedures and MAKE files to automate the porting process and to provide a comfortable environment for development and test. Some real problems appeared on the PC. One problem was the incompatibility of several software packages which could be discovered only by trial and error. Another problem were compiler errors especially in the runtime libraries. The biggest problem was debugging. Due to storage constraints on the PC (the $640 \mathrm{~KB}$ boundary) and the amount of storage used by the NOS Kernel it was "a little bit" awkward to debug RSC.

In summary, it can be said that the engineered design of the DAC NOS in conjunction with the Portability Guidelines and the dependent file concept has demonstrated to be well suited to achieve the portability objective.

\section{Performance}

One of the goals of the NOS Kernel was to achieve good performance though it was not our main goal. By experience communication over networks is expensive. Therefore RSC was designed to minimize the number of protocol messages sent.

Only preliminary measurements of elapsed times have been obtained so far, there is up to now no detailed analysis of where this time is spent. We have measured the execution time of a service request cycle, i.e. a carrier is sent to another node, accepted and inspected there, and immediately returned to the sender who waits for the carrier. Measurements were taken during regular daytime with medium load on the hosts. First we measured three different "local configurations", i.e.

- two RSC entities on one IBM/370 (type 4361),

- two RSC entities on one DAC VAX (type 8600),

- two RSC entities on two PCs (type AT) on a token-ring.

Then we examined a remote interaction between

- two IBM/370 computers (4361 and 3083), connected by a $64 \mathrm{Kbps}$ line and IBM internal transport protocols,

- two VAX VMS computers ( 8300 and 8600 ), connected by an Ethernet LAN and DECnet protocols,

- an IBM PC-AT and a VM on an IBM 4361 connected by a token-ring and IBM internal transport protocols.

The following table shows the results for a basic two-way request cycle: 
REQ. CYCLE VM to VM VMS to VMS PC to PC PC to VM

$\begin{array}{lllll}\text { local } & 50 \mathrm{~ms} & 200 \mathrm{~ms} & 165 \mathrm{~ms} & \ldots \\ \text { remote } & 125 \mathrm{~ms} & 500 \mathrm{~ms} & \ldots & 345 \mathrm{~ms}\end{array}$

For the homogeneous VM to VM case and the PC to VM case we measured also the elapsed time for a Read-Window operation for various window sizes. These investigations have shown that for VM to VM the local (remote) execution time contains a fixed portion of 45 (112) milliseconds. For PC to VM we measured a fixed portion of $315 \mathrm{~ms}$. Clearly, there is also a window size dependent part that is determined by the data transmission speed and the overhead to handie internally several transport system buffers. The following table shows the results for the three configurations:

\begin{tabular}{lccc} 
READ_WND & VM local & VM remote & PC to VM \\
\hline 100 bytes & $45 \mathrm{~ms}$ & $112 \mathrm{~ms}$ & $315 \mathrm{~ms}$ \\
$10 \mathrm{~K}$ bytes & $110 \mathrm{~ms}$ & $1410 \mathrm{~ms}$ & $600 \mathrm{~ms}$
\end{tabular}

The differences in these results reflect the various processing capacities of the involved computers, the diversity in the underlying transport hard- and software and the efficiency of the implementations of KSC on the three operating systems.

\section{Summary}

The NOS Kernel is still under development. Its interface is at a comparatively low level. Library functions for Service Calls could provide more ease of use for the programmer. As time evolves a toolbox will arise containing a set of higher level objects and operations based on the RSC low level interface. This will provide the flexibility for different levels of distributed applications. Open research problems are the introduction and handling of replicated objects, and the support of transaction processing. Further, portation to UNIX and to the new PC Operating System OS/2 are envisaged.

The goal of RSC was to provide a network transparent interface for the development of distributed applications and system services. The application programmer should not be burdened with heterogeneity and distribution aspects. The NOS Kernel, as it stands now, runs as a compatible extension of three very different operating systems without affecting local applications and services thus meeting its transparency and coexistence objectives. 


\section{System Management Services}

As part of the DAC NOS we developed a number of management services, thought of as a basis for the various applications in achieving things such as location independence, transparency, security, or charging for resource usage. Two of these services have been implemented yet, others may follow in the future.

The two services realized so far are a Directory Service (DS), and an Authentication/Authorization Service (AAS). Both have faced us with a number of problems, and a set of possible solutions for them. Providing some insight into the problems encountered, the alternatives chosen, and the experiences gained from these choices, is the intent of the following pages.

\section{Directory Service}

From the very beginning of the work on the DAC NOS it has been realized, that the system requires a component capable of maintaining, distributing, and protecting information about network resources and services. Beyond this obviously very general statement there only were three rather abstract service descriptions, that could have been used as a guide during the design process. According to these service descriptions the directory system should provide for:

- Name-to-property binding ('White Page Service'),

e.g. Name-to-address binding.

- Property-to-set-of-names binding ('Yellow Page Service').

- Name-to-set-of-names binding.

In addition a number of client controls have been anticipated. One of them, for example, should allow clients to restrict the extent of searches for required information, thereby limiting the costs of information retrieval. Before giving, however, details of our solution, let us first consider some of the central issues we found ourselves concerned with during the design.

\section{Design Issues}

When devising a service for a system such as the DAC NOS one is inevitably compelled to a distributed solution. Having accepted this fact for the directory system, there immediately raises the question of naming. Clearly, each object in the system must be named. Global names being valid everywhere in the system must be unique to avoid ambiguities; they should further be user oriented, so that each name calls forth an association with the object it refers to. While naming is a crucial problem in each distributed system, it becomes even more difficult in a heterogeneous distributed environment. 
Another issue of great importance pertains to the applications being supported. The choice here is between a simple static concept supporting a predetermined number of well known services, and a more complex dynamic solution providing for the support of any conceivable current and future application.

A question closely related to that of supported applications concerns the data model. Each object offered in the directory is represented there by a self-contained directory entry. The structures, contents, and relations of respectively between these entries determine the data model of the directory system.

In a distributed system performance may greatly be enhanced, if essential information is replicated, i.e. kept in multiple instances, so that access distances can be minimized. Distinct forms of replication mainly differ in the way contents changing accesses are performed. For us it was enough to concentrate on copies on one hand, and cached entries on the other. While copies always are kept consistent with one another, this is not true for cached entries. Cached information may be out of date, and therefore useless.

Protection has been introduced as one of the central topics of the directory system. Protection here is meant as controlling access to information kept in the directory. The main question that needed to be answered in this respect was that aimed at an appropriate granularity of access control. Should access rights be granted on a per entry or on a per attribute base. Granting rights on a per entry base may be disadvantageous, if more than one application makes use of a single object type. Granting rights on a per attribute base may be unhandy, and can drastically degrade performance.

The way, clients can request services is probably of great influence on the acceptance of the directory system. The possible alternatives we met here were a language based client interface supported by a specially devised query language, and a procedural approach founded on a elaborate presentation facility of RSC.

Beyond requesting information about a single object, we felt the need to extend information retrieval into the direction of a selection and orientation facility. Suppose, you want to do a job, but apart from being done at all, you want the job being done at best with respect to some given criterion. Then it's obviously not enough to get information about single objects. Instead the directory must provide facilities that allow for the selection of an object (e.g. a device) according to that higher level optimality constraints.

Let us now briefly present some of the solutions we have adopted in our concept of a directory system.

\section{Design Decisions}

Very soon during the design we realized, that names are merely a special kind of resources, that require proper management. As a result of this recognition we devised the directory service as consisting of two subservices; one, which we called the name service, in charge of name management, the other, which we termed the entry service, responsible for all activities being pertinent to the maintenance and use of the directory database. 


\section{Name Service}

We decided to realize a two level highly structured name space. Global names obey the following general structure: < global_name $>::=<$ domain_name $>$.<local_name $>$. The first part denotes the naming domain, and is composed of a sequence of components that mirrors its site in the hierarchy of domains. With respect to the local name part, the name service allows for the establishment of local, type specific naming conventions which we call nameforms. The management of names proper is accomplished by a set of operations to take, generate, and release names.

Naming domains can also be shared. Suppose, for example, in a naming domain A there is the casual need for using names that actually belong to a naming domain B. At the same time clients in B should not be allowed to use names belonging to $A$. This can be achieved by establishing a directed connection from $\Lambda$ to $B$, which allows for the sharing of naming domain $B$ between the clients of $A$ and $B$. Clearly, such a directed connection may only be established as a result of a bilateral agreement between the administrations of $A$ and $B$.

\section{Entry Service}

The perhaps most significant decision pertaining to the entry service has been our renunciation of information replication in the form of copies. Operations modifying the directory database can only affect the part resident in ones local domain. All activities pertinent to information retrieval have been delegated to a separate Orientation and Selection Service (OSS), acting as an ordinary client of directory servers. With this service clients can restrict the scope of their searches by setting an appropriate horizon. For the information retrieval proper, we have relied on a content oriented solution instead of a name directed approach.

Directory entries are typed entities consisting of object name, entry owner, and a set of type specific attributes. We have provided for off-line definition, extension, restriction, and cancellation of types to adapt to new applications, and to cope with changing requirements of existing ones. Access control is performed on a per attribute base using access control lists. Modification of an entry's access control lists is reserved to the owner of the entry. To accelerate repetitive access to single entries, caches are maintained as part of the directory servers. Management of these caches is done by the Orientation and Selection Service.

As to the client interface we have decided to follow the procedural approach. Operations to insert, read, modify, and remove directory entries can be requested by calling the respective procedures.

\section{Experiences}

Before ending with our discussion of the Directory Service let us report some of the experiences that we have gained with its design and realization, and as far as already possible its usage. 


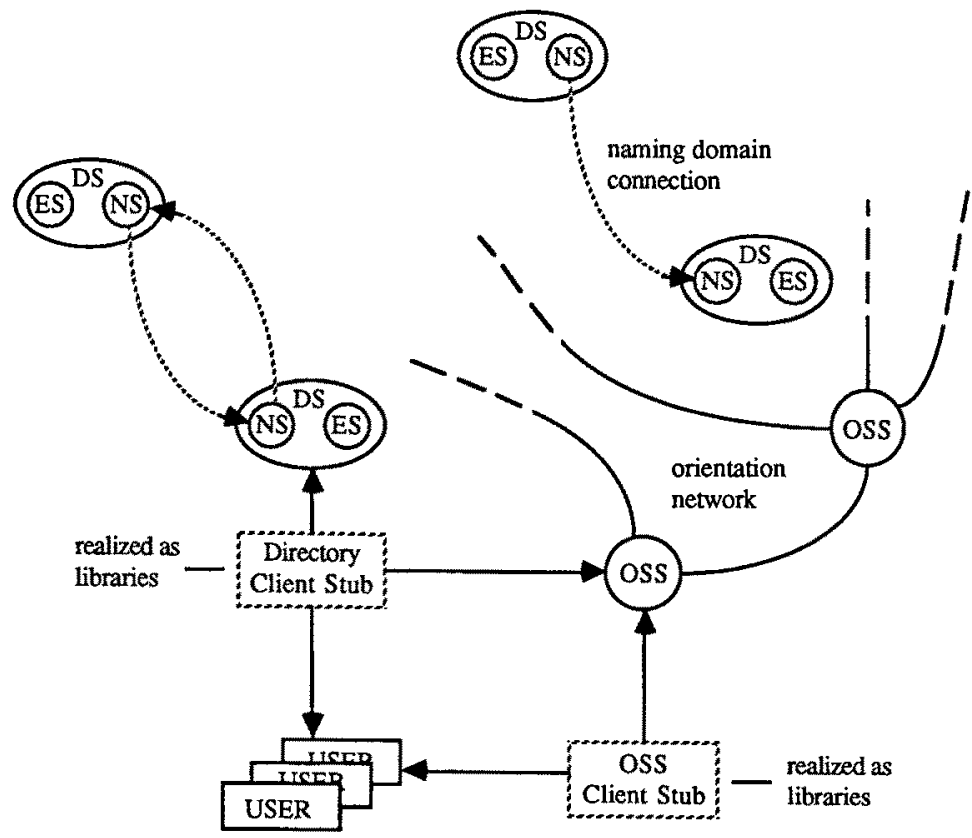

Figure 4.1. Structure of the DAC Directory System

(1) Naming has shown itself as being the central management issue in the DAC NOS. Many services are closely related to naming, and their ultimate organization is highly influenced by the solution adopted for name management.

(2) Contents oriented search using flooding has proved to be a flexible and efficient approach to the information retrieval problem. With this approach query operations are broadcasted among OSS servers along a logical network, which we call the orientation network. In contrast to this, with a name directed approach each name must serve as a guide to the location where its corresponding entry may be found. It is, however, not clear how a Yellow Page Service should be realized with this approach.

(3) Our refusal of information replication greatly hampers selfadministration of the directory system. For selfadministration to be applied the directory must hold information about its own configuration as part of the directory database. Such information for being useful, for example in query routing, must be highly replicated under consistency constraints.

(4) The decision to use server based instead of client based caching allowed us to implement client stubs solely in the form of libraries; no resident code is required for them.

(5) As regards the data model typing has shown to be advantageous for it impresses a structure on the directory database. Clients now know what they may query for, because all objects of a certain type are described using the same set of attributes. As the number of supported types grows, however, operations capable of revealing the structure of types may be required. 
Providing only for off-line alteration of the directory (database) structure has proved tolerable, for such alterations occur fairly rare.

(6) We already stated, that access control when performed on a per attribute base must be defined with care. Default settings for access rights must be selected in such a way, that the need for explicitly specifying access control list remains to be the exception.

(7) Procedural client interfaces have shown to be superior to language based ones. With an early version of a directory server we devised a special language for request formulation, as an attempt to cope with the problems of the client interface. The main difficulties we met with this approach were: embedding of service request into C-programs, performance degradation due to the need of parsing the request in each server, and returning query results to requesting clients.

(8) RSC has shown to be a powerful tool for writing distributed systems; but for being honest we must state, that we only required a small subset of its actual capabilities, namely: processes, two way messages (carriers), eventlists, locks, and presentation facilities. We missed, especially when implementing the flooding algorithm, an RSC primitive for carrier oriented broad- or multicast.

(9) The decision to realize the OSS as an ordinary client of directory servers has shown to be a bad one. OSS must be regarded as an integrating top layer of the directory system and not as a service of its own right.

\subsection{Authentication/Authorization Service}

Let us again start the presentation with the list of general requirements, that stood at the beginning of our work on the Authentication/Authorization Service (AAS). According to these requirements the AAS should:

- Provide for mutual identification of interacting partners.

- Assist application services in controlling access to their objects.

- Close the security gap introduced by free accessible workstations.

As with the directory system these requirements led us to a number of design issues whose most essential ones will be described subsequently.

\section{Design Issues}

In the following our system is considered as consisting of a subject universe, an object universe, a set of access rights, and an access relation. Subjects are the active elements of the system. Each subject is uniquely determined by a number of characteristics combined in a subject description. Objects are the passive elements acted upon and defined by the various applications, as are the access rights for these 
objects. The access relation is realized in the form of access control lists. With respect to this model the following questions have called for answers.

- What should be regarded as a subject ?

- How to describe subjects and where to keep the subject descriptions?

- Who maintains and keeps access control lists ?

In a distributed system the subject universe can become vast. Thus for keeping access control lists reasonable in size, subjects must be collected into groups. This immediately raises the question of desirable group models.

Again we found ourselves concerned with the problem of naming; this time, however, with the reusage of names. Suppose a user, let us call him user A, is registered in the system and his name is widespread as an element of memberlists of groups. If our user some day leaves, his subject description will be removed, but not the occurrences of $A$ in the groups' memberlists. $A$ is a frequent name; so sometimes in the future another $A$ will become a registered user of the system. If no provisions are made, this new user $A$ takes the identity of the former $A$ with respect to authorization. This is clearly not the way we want the things to behave.

A necessary precondition for access control is authentication. By authentication we mean the verification of a purported identity. The main problem here was, how and where such a verification should take place, especially in the context of free accessible workstations. With a timesharing system there is always some control over the set of possible users, for they all are registered in the system itself. This is not true for workstations. Everyone having accession to such a device can use it and therefore in the DAC environment can obtain access to services and programs residing on a host.

\section{Design Decisions}

We rely on a password based authentication scheme. Subject descriptions are kept in the directory and maintained via the directory client interface. A user that wants to identify himself to the DAC NOS specifies apart from his name and password an AAS server capable of performing the authentication. If the specified server is unknown to the user's local AAS server its address is obtained by a OSS request and the two servers apply an internal authentication protocol for their mutual identification. The user's authentication request is then transferred to the specified server which tries to verify the purported identity. The result of this verification, either success or failure, is returned to the local server together with the user's group list in case of success.

Authorization and maintenance of access control lists lies in the responsibility of the respective application servers. Access rights can be granted to groups only never to a single subject; so all names appearing in access control lists must be group names. 


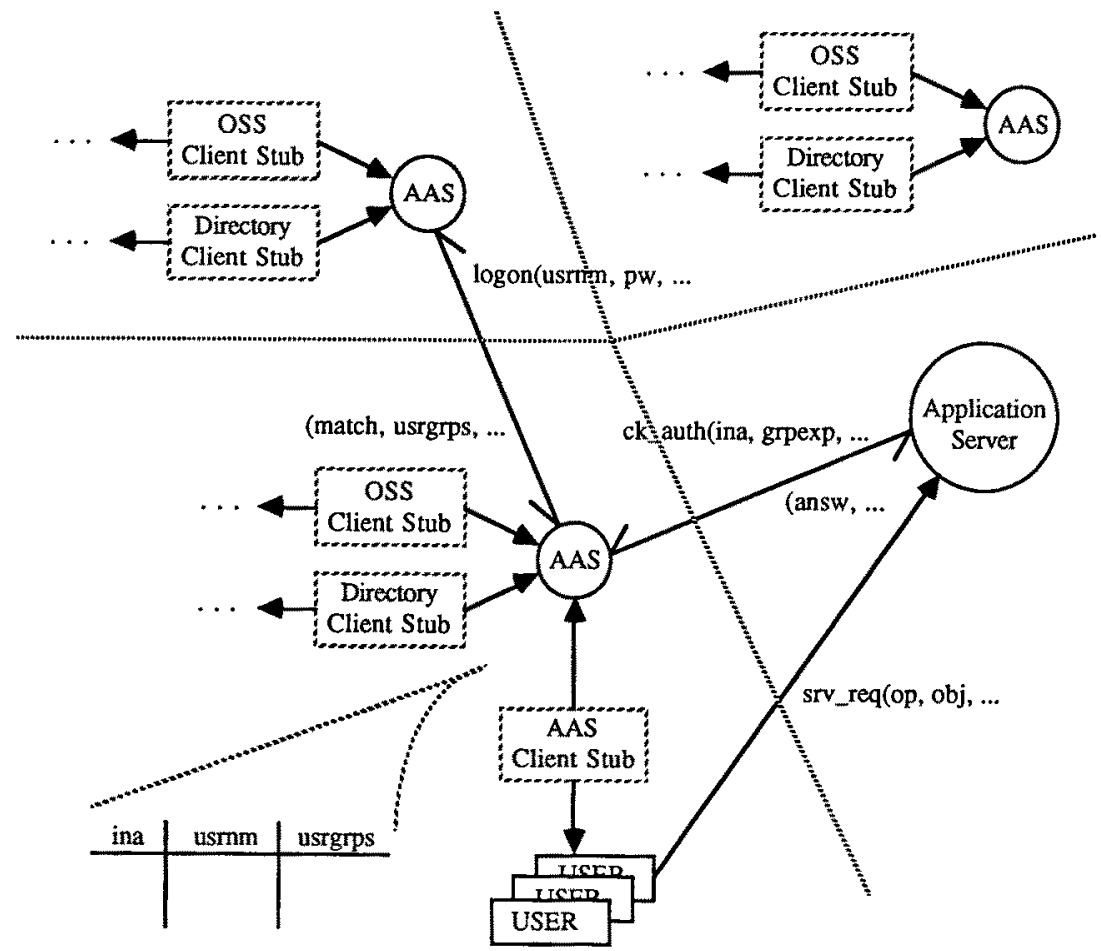

Figure 4.2 Structure and use of the DAC Authentication/Authorization System

The problem of name reusage has been solved by internal use of object identifiers only. Each usage of a name is mapped to a unique identifier. Uniqueness thereby is meant to be a time-independent property; two successive uses of the same name are always mapped to different object identifiers.

Suppose a user, requesting an operation op on an object obj from an application server (e.g. a file server, or a mail server). Knowing the access requirements of op the application server builds a group expression from the access control lists of $o b j$, that the user must fulfill for having its request being served. This group expression is transferred together with the request's source address (user address) to the user's local AAS server for authorization check. Doing so requires that AAS servers are bound to a fixed socket-address in each subnet (domain). The result of the authorization check is returned to the application server indicating, whether the service request should be accepted or refused.

\section{Experiences}

Let us again report some of the experiences that we have gained with the design and realization of the Authentication/Authorization Service.

(1) Internet addresses are protected entities in the DAC NOS and serve completely as subject creditals. Other forms of creditals are conceivable and may be used in later versions without major changes of the principal service structure. 
(2) The directory has shown to be the proper location for keeping long term protection relevant information. Due to performance reasons this statement is not valid for short term information such as the address/name - correspondence tables kept by the AAS servers.

(3) Information cached in AAS servers (e.g. the users' group lists) should be updated periodically in order to react as fast as possible to changing authorization patterns.

(4) Recursive group definitions with automatic expansion would be desirable. Such a facility, however, bears the problem of cyclic definitions and therefore requires distributed methods for cycle detection.

\section{Remote File Access}

Remote File Access (RFA) is a distributed file system for heterogeneous networks. The objectives of the development of RFA were twofold. The primary objective was to make access to remote files as convenient as possible. The second objective was to act as complex testcase for the completeness and adequacy of the services offered by the NOS kernel.

RFA has been designed and implemented in parallel to RSC. The design considerations and the user interface of RFA have been published in a different paper [12]. Here we will give only a short overview on RFA, and then discuss the system requirements of distributed applications in heterogeneous networks. The major part of this chapter deals with the experiences gained during the development. Topics discussed are the achievement of the transparent access to global files, the design for portability and scenarios for the usage of RSC objects. The chapter finishes with closing remarks on RFA and the NOS kernel.

\subsection{Overview of RFA}

RFA is a global homogeneous file system for heterogeneous networks. The file naming structure is hierarchical; the running prototype supports sequential record oriented files. The RFA file system is partitioned into multiple RFA file servers, each being responsible for a subset of RFA files.

RFA Servers use the local file systems of the "underlaying" local operating systems. This technique minimizes the effort needed to port RFA servers to different operating systems. It also allows to store the data of global files in local files, thus allowing easy exchange of files between RFA and the local file system and easy maintenance of global files through local utilities.

RFA users run the RFA Client software, which is an extension and - optionally - a modification to the local operating system. The extension offers access to the global RFA files through a procedure and a command interface. The modification opens the local filesystem interfaces for the global RFA files. Sets of global files can be bound ("mounted") into the local file system as "virtual volumes". A table driven file name translator associates local alias names to global file names. Global files can be accessed 
transparently via their local aliases in the same way as local files. Existing application programs can use global files without any change.

Figure 5.1 shows a scenario with two users having access to RFA files. On top, there is a RFA file server UKA maintaining a set of RFA files - named UKA.DAC02. This set contains three files with the global names: PROGR.PASCALSOURCE, TEST15.DATAREAL and TEST16.DATAREAL.

Lower at the left hand side, a PC user accesses the file set UKA.DAC02. The users has attached the set using the RFA command 'AFS UKA.DAC02 as b' as a local disk drive with name b. Upon accessing the file set, the files contained got local alias names according to the syntax of PC DOS. These names are derived from the global names by RFA applying user specified rules as shown in the box above. In this case the local alias names are PROGR.pas, TEST15.dtr and TEST16.dtr. (The last of is not shown due to the limited space for the figure.) With the construction explained above, the PC user can access the file of the global file set through their local names; in the figure the users calls the unmodified local editor pe to edit the file PROGR.pas, i.e. the global file PROGR.PASCALSOURCE. The right hand side shows a similar situation for a CMS user. In both cases the remoteness of the files remains completely transparent to the application programs.

\subsection{Requirements to the NOS Kernel}

\section{Service Access Points}

Consider the situation in a local file system: If a user wants to access the data of a file, he asks the file system to prepare the file for the access, i.e. to "Open" it. The local file system returns an identifier, an "open file handle" to the user. This handle serves as access point for further access operations. In a distributed system a similar kind of service access point is needed. It should be service specific and independent of the number and identification of the serving processes. Thus, a construct for a service access point should be available to represent services - like file servers - or objects - like files - within the network. This construct should have similar characteristics as the "open file handle" to the user. It can be passed to other, possibly remote processes.

\section{Bulk Data Transfer}

As the response time of remote requests depends more to the number of data transfers then on their size, bulk transfer is a "must" from the point of view of efficiency. But clients may want to access remote data in larger or smaller portions; while servers prefer to handle larger portions to reduce their overhead. The communication system in between should handle the transfer of data assembled into large blocks. It should accept portions of data of arbitrary size for transfer and should allow to receive the data in portions of different size, i.e support assembling and disassembling of blocks of data. 


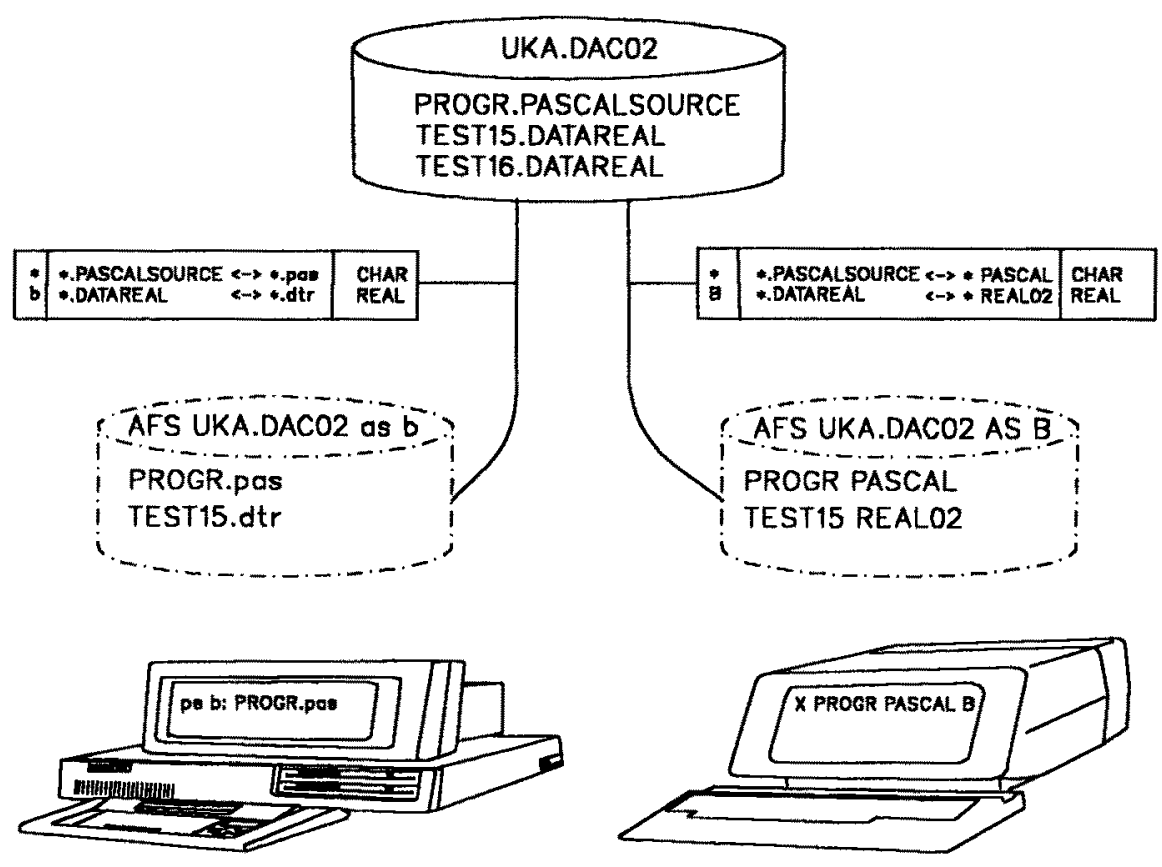

Figure 5.1 Using RFA

\section{Presentation}

As the DAC network is heterogeneous, the presentation of data is of concern to any network application. RFA control messages are internal to RFA and cause no special problems. The exchange of file contents does: To convert data from one local coding to a different coding, the type definition has to be known. Given the type definition for the records of a file, the communication system should be capable to transfer bulks of records with a single request and recode the data from the local coding of the sending site to that of the receiving site. This is a prerequisite for the transparent handling of global files in a heterogeneous network.

\section{Directories}

Any file system maintains a catalogue of the contained files. The catalogue keeps file specific information for the orientation of the users and for file system purposes: file name, attributes, internal file system information. In a distributed system, Directory Servers keep databases of netwide accessible information. Directories can be used to keep the file catalogs. It is a matter of efficiency whether and how much catalogue information is kept locally be the file server itself. For the orientation of the users, the directories should at least contain the file server names and the names of the file sets. 


\section{Access Control}

Files are globally accessible objects needing access control. It should be possible, for example, to allow the appending of file records to a group of users, but not the overwriting of already written records. Thus, the access control mechanisms should be flexible enough to handle application specific access rights. It should work on a basis of Unique Network User Identifications.

\subsection{Experiences with the Design and Implementation of RFA}

\section{File Access Transparency}

The realization of file access transparency, i.e. the access to remote file via local interfaces, has two basic requirements: The ability to intercept the service calls to the local file system and a mapping of the service calls, of the file names and of the file contents from the local file system to their semantical equivalents in the global system. The interception techniques used for RFA will be discussed in the following section. The service calls and return codes can be mapped straightforwards provided that the global system interface is flexible enough. The mapping of local to global file names and vice versa is a slightly more difficult issue; it will be discussed in a subsequent section.

\section{Intercepting Service Calls to Local Operating Systems}

The RFA Client software needs to get control on each service call to the local file system to check whether the call is directed to a remote file. These calls are intercepted and rerouted into an extension of the local operating systems kernel. There are mechanisms for such extensions in VM/CMS, PC-DOS and VAX/VMS. The interception, essentially rerouting of service calls is supported in PC DOS. There is a comparable interface in VM/CMS; the supervisor call to CMS uses a transfer vector pointing to the addresses of the CMS kernel functions. As CMS is a single user system, the addresses can be replaced to point to RFA code. Some CMS kernel functions call each other directly without respecting the entries of the transfer vector. Modifications to these routines had to be done. VMS also uses the transfer vector technique. However, as in VMS any process uses the same copy of the operating system, the vector entries can only be changed for all users or none. As alternative, a device driver interface can be used. With this technique, any request to a specific device is routed by VMS to a dedicated device driver.

The interception mechanisms for PC DOS and CMS are completed and in use. The efforts to achieve transparent access to remote files in VMS is still under way.

\section{File Name Mapping}

Global file names of RFA are segmented names with only a length restriction of currently 64 characters. Usually local file names have more syntactic restrictions. 
The mapping process consists of two steps. At the binding of a set of global files, a local alias is given explicitly to the common prefix of a set of global files. The suffix of the file names is generated by a rule driven translator. A YACC generated parser [13] compiles the translation rules into an internal data structure which is used by the translator to derive local from global file names and vice versa. Further details may be found in [12].

The usage of the Unix YACC tool gave us flexibility in the rule syntax because parser routines are generated automatically from syntax definitions in an extended Backus Naur form.

\section{The Components of the RFA Software}

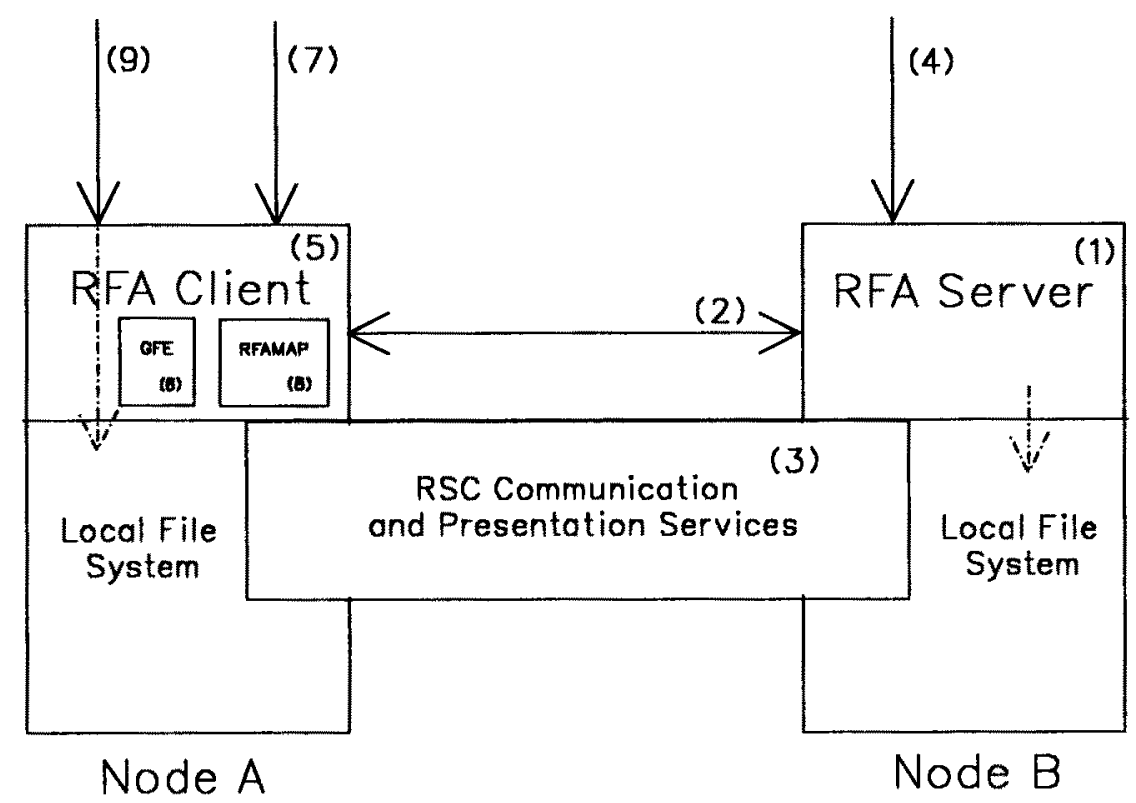

Figure 5.2 The Components of RFA

Figure 5.2 gives an overall view of the components of RFA: File servers (1) provide the storage for files and offer a homogeneous global interface (2) via the communication system RSC (3). Privileged users may control the file server via file server commands (4). File servers are dedicated logical nodes: workstations, virtual machines or "heavy-weight" processes.

RFA-Clients (5) are extensions of the local operating system. The Global File Environment (GFE) table (6) describes the association of global file set names to local file sets, i.e. local virtual volumes. Some new commands (7) are used by the client to associate a global file set with a local file set name and thus control the contents of the GFE table. The RFAMAP table (8) contains the active set of rules used for the translation of file names. The GFE table and the RFAMAP table together make it possible, 
that application programs can access and operate on global files via the unmodified interface of the local file system (9).

\section{Portability}

Generally there is a trade-off between portability and efficiency. Portable software tends to be inefficient because more efficient, system dependent interfaces are not used. In RFA, some modules of the software are portable, others are not. Some of the non-portable modules can easily be adapted to different interfaces with a few changes.

- The attachment of the RFA Client software to the local file systems is not portable.

- The client and the server commands follow the syntactic conventions of their local host operating system; they are not portable, but adaptable.

- The interfaces to the communication system and the internal mechanisms of the RFA clients and servers are portable.

- The servers interface to the local file system is portable if there is a robust and efficient run time library for the implementation language. For CMS and VMS more flexible and more efficient system dependent interfaces are being used. Thus, this component is not portable.

Several modules are being used within both, the client and the server.

\section{The Usage of RSC Objects and Mechanisms.}

\section{RFA Server Ports}

In RSC, ports conceptually define typed resources that accept a limited and well defined set of request types, i.e. the structure of the acceptable carrier is defined for each port. This includes the type of the "send" and "return" messages and the number and type of the inserted objects (windows, etc.). Thus, the offered service is encapsulated into an abstract data type.

Following this conceptual view, the RFA server offers a "main port" for opening files, for catalog inquiries and file maintenance. A different type of port is used for the access to the contents of a file. This type of port accepts requests like "read", "write" or "close". The open processing of the file server creates an open file port and shares it with the caller. Subsequently this port is used as service access point for such requests as "read" or "write". The port is deleted when the file has been closed.

The mapping of open files to a port and a separate RSC process for serving the port has several positive consequences. Some of them will be given in the sequel. The file server needs not to be concerned with dispatching. If multiple requests queue up, $\mathrm{RSC}$ contains the mechanisms to ensure a fair distribution of the file service among the clients. Authorization checking takes place during open time. At this time, the port is only shared between a client and a server. At subsequent calls to the port, the server can rely on the authorization of the caller. It is easy to add file service specific accounting to a server. Since the 
service providing process runs under the account of the client, this process can be charged for any desired account units. RSC and the accounting server collect the bills for the client process.

\section{RFA Bulk Data Transfer}

Bulk data transfer is implemented via RSC window objects. In principle, two variants of window usage seem to be possible:

1. The window is created over the clients data buffer. The Clients offers a buffer to receive information or file data from the file system. The RFA client software creates a RSC window over that buffer to allow the server to write into that area of client storage via the RSC "write window" function. This variant has the advantage, that the server buffers data only for the duration of a read of write request. Across these requests, a minimum of information is kept at the server for an open file.

2. The window is created at the servers site. As a result of a service request, the server has filled a buffer with information. A RSC window is created over this buffer to make it available to the client via the RSC "read window" function. This variant has the advantage, that multiple clients can read from the same buffer if they have requested the same information. It has the disadvantage that the server has to keep buffers for his clients until the clients release them. If the client is not able to do that, server resources are blocked.

The first variant has been implemented to minimize 'housekeeping' at the servers site. During the development of the presentation system and the portation from CMS to the PC the second approach looked more and more interesting.

Looking deeper into the first variant shows the reasons. Having established a 'dedicated' file port, the client sets up a carrier and a window to read some data from the file. The carrier is sent to the server. The server accepts the carrier, reads the file data into a private buffer and then writes it into the clients window. Some speed improvement in the processing can be gained if the server is able to buffer the complete file as long as the file is open. Then, the server simply calls "write window" with the appropriate parameters to copy data from its buffer into the window of the client. Thus, the server activity is only needed to move data into the window. Now the advantage of the second variant can be seen: RSC can do buffer access if the window is on the servers site without any activity of any server process. The server creates a window over the buffered complete file. It shares the window with the client by putting it into the reply carrier instead of the dedicated port. Then, the window is accessible for the client. It can read data from the window at any size from arbitrary window offsets. No server activity is needed until the file is to be closed. The server closes the file when the carrier and the window has been returned. The event can be noticed at the servers site, and triggers the servers close processing. Sharing of the file window between multiple clients is possible. Following this approach no port is necessary to access the data in the open file. This approach can be characterized as data oriented in contrast to the activity oriented first approach.

With the original definition of RSC, the second approach was not viable. RSC has since been changed to allow for the second approach. The format description of a window may be determined any time 
before the first usage of the window. Thus, there is a choice between the two approaches; its a trade-off between efficiency and implementation effort.

There is one more aspect in finding a choice between the two approaches: During the data transfer for the carrier messages or for window data the transformator of the presentation system converts the coding of the data. The transformator is controlled by the type definitions. The type definitions may define composed objects as sequence of basic objects (scalars). Possibly, the data transfer has to be stopped during the recoding of such a composed object because some buffer is filled. The transfer has to be resumable without loss of information. Therefore, the transformation state is kept in such situations and used as the transfer is resumed. This transformation state is attached to the RSC object being transferred, most frequently a window. This means that the window object may not be deleted as long as such a transformation state is attached to it. For the first approach of having the window at the clients site, this means, that essentially the same window has to be used while the file is open. RSC window attributes have to be changed to 'move' the window over the users buffer.

There are operating systems that map complete files into a segment of virtual memory [14]. For these systems the second approach becomes even more interesting. In general, the second variant seems to be advantageous in special cases: small fles and large buffer spacc.

\subsection{Summary}

In this chapter it has been shown, that RSC is an adequate base for complex applications like RFA. The interfaces to the file server can be limited to the functional aspects of the application. There is no need to design protocol elements for authorization, accounting, node failures, time outs or any other aspect of remote communication. This makes the interface design much easier and increases its stability. Handling differences in data representation between different machines has found a solution with the association of data descriptions with Ports. The presentation functions of the NOS kernel are flexible enough to handle headers or trailers of variable length records transparent to RFA. An important aspect of any network file service is the efficient transport of large volumes of data. Such services are often supported with separate bulk transfer protocols. With RSC this is of no concern to the application. The RSC 'read window' and 'write window' operations use a bulk transfer protocol whenever it seems to be appropriate. The window object is thus not only convenient, it also allows for efficient implementation. The distribution of file services over file servers in a dynamic user environment is unlikely to remain stable. With the sharing capabilities of RSC, a redistribution of services to servers may remain transparent to the clients.

RFA is a running prototype for distributed file systems in heterogeneous networks. With RFA users of CMS and PC DOS (later also VMS) get transparent access to remote files anywhere in the network without taking care for heterogeneity. 


\section{Conclusion}

With the emphasis on resource access transparency and the local system paradigm we have set ourselves ambitious objectives. It was clear to us from the beginning that the resultant NOS will be fairly complex, although masterable. Nevertheless, the parallel development of such a system for three completely different operating systems would clearly have been beyond our scope. As a solution we designed for portability. The components of the NOS are first developed on a specific system and then ported to other systems.

Today we can say, that our primary goal has been met. We were successful to build a portability environment on the three target systems with moderate effort. The portability of the complex components on this basis proved to be excellent. Today we have the prototype of a Network Operating System for three widely used operating systems supporting transparent resource access and the development of portable applications. The NOS can be adapted to further operating systems with a reasonable and, by comparison to the total development even small effort, in the order of person months per component.

The most important elements, which contributed to the obtained solution were, in our view, the following:

\section{Few basic concepts and guidelines}

The local system paradigm proved not only as an objective, it was extremely helpful in making design decisions early and in a consistent way. It helped us after few deviations to determine access controlled object sharing as the proper analogue to the shared memory of local systems. It helped us also, to design the proper protection and resource usage monitoring facilities into the NOS-Kernel. And, it guided us to design a systematic approach to transparent remote file access, which in turn may be considered a prototype for the transparent access to other types of remote resources.

\section{Proper layering of interfaces}

During development it turned out that the layering of interfaces in figure 1.1 was essential. Each interface played a clearly defined role within the overall system. It was possible to define these interfaces early in the project and to keep them stable. This simplified the development of the NOS by three remote groups with infrequent communication possible. For example, it was possible to design and develop the System Management services based solely on the NOS-Kernel interface. 


\section{Avoid too simple approaches}

Simplicity is a generic objective with very wide applicability. But this objective may also, if misunderstood, lead to pitfalls. We believe, that building the NOS on a simple message based interface, would in fact, have made the total NOS more complex. Code, that is now centralized in the Kernel (and 100 percent portable) would have had to be reproduced in many components and applications.

A further example of a possible pitfall which we avoided, is to ignore the importance of compatibility and coexistence. This made the development of the lower layers more difficult, but in retrospect, coexistence has helped us during development significantly. NOS applications and LOS applications can be freely mixed. Thus all tools available on the LOS were available not only for development, but also during debugging and testing. The extra effort for coexistence brought thus already a pay-off during development.

\section{Final remark}

DAC NOS, as presented here, is a prototype providing a systematic way for the accommodation of heterogeneity. As a prototype, it is certainly not complete in all functional areas. However, we can say, that the approach is feasible and its usefulness can be evaluated by users. In our view, the approach is now developed to an extent, in which the development of standards for a generally accepted Network Operating System has a sound experimental basis.

\section{Acknowledgements}

The work reported in this paper is the joint achievement of the authors and their colleagues H.v.Drachenfels, H.Eberle, K.Geihs, M.Salmony, M.Seifert, P.Silberbusch, M.Wasmund, G.Wild, C.Foerster, R.Oechsle and M.Zöller.

\section{References}

1. D.R. Cheriton: The Thoth System: Multi-Process Structuring and Portability, North Holland, Computer Science Library No. 8, 1982, ISBN 0-444-00701-6.

2. R. Staroste, H. Eberle: Kernel Service Call, IBM European Networking Center, Technical Report TR No. 43.8701,1987.

3. M. Salmony: Experiences in the design of a transport system for Heterogeneous Environments, $I B M$ European Networking Center. Technical Report TR No. $43.8601,1986$.

4. H.Eberle, A.Schill and B.Schőner: Beobachtung bei der Portierung von $C$ Programmen von /370/CMS (portable C) zu PC DOS (microsoft C) und zu VAX/VMS, IBM European Networking Center, DAC Technical Memorandum No. 26, 1987 (in german, available from the authors).

5. IBM Corporation: IWS/8088 Development Workbook, IBM T.J. Watson Research Center, Yorktown Heights, N.Y., Internal Report .

6. N. Carriero and D. Gelernter: The S/Nets Linda Kernel, ACM TOCS Vol.4, No.2, pp.110-129 (1986).

7. D.R. Cheriton: Problem-oriented Shared Memory: A Decentralized Approach to Distributed Systems, in Proceedings of the 6th International Conference on Distributed Computer Systems, IEEE (1986).

8. J.A. Stankovich, A Perspective on Distributed Computer Systems, IEEE Trans. on Computers, Vol. C.-33, No. 12.1984, pp.1102-1115 
9. H. Eberle, K. Geihs and M. Seifert, Remote Service Call: Object and Operation Reference, IBM European Networking Center, DAC Technical Memorandum, No.16, (1985) (available from the authors).

10. B. Liskov et al.: Limitations of Remote Procedure Call and Static Process Structure for Distributed Computing, MIT Programming Language Methodology Group. Memo 41 (1985).

11. G. Wild and M. Zöller: Darstellungsproblematik fuer heterogene verteilte Systeme, in Proceedings of the GI/NTG Conference "Communication in Distributed Systems", Aachen/W-Germany (1987) (in German).

12. U.Hollberg, H.Schmutz, P.Silberbusch: Remote File Access: A Distributed File System for Heterogeneous Networks, in Proceedings of the GI/NTG Conference "Communication in Distributed Systems", Aachen/W-Germany (1987) (in German). ENC Technical Report No. 43.8611, Heidelberg, W.-Germany, 1986, available from the authors

13. St.C.Johnson: YACC: Yet Another Compiler-Compiler, pp. 353-387 in: "UNIX PROGRAMMER'S MANUAL", Bell Telephone Laboratories, Incorporated, Murray Hill, New Jersey 07974, Holt, Rinehart and Winston, ISBN 0-03-061743-X, 1983

14. IBM Corporation: AIX Operating System Programming Tools and Interfaces, IBM Corporation, IBM Advanced Interactive Executive Operating System, SV21-8010-0, 59X9111, 1985. 